\title{
Investigating the Quality of Spiess-Like and SPSA approaches for Dynamic OD Matrix Estimation
}

Xavier Ros-Roca ${ }^{\mathrm{a}, \mathrm{b} *}$, Lídia Montero ${ }^{\mathrm{b}}$ and Jaume Barceló ${ }^{\mathrm{a}, \mathrm{b}}$

${ }^{a}$ PTV Group, Karlsruhe, Germany; ${ }^{b}$ Statistics and Operations Research Department, Technical University of Catalonia, Barcelona, Spain

Xavier Ros-Roca (Department of Statistics and Operations, Technical University of Catalonia, Campus Nord, Building C5, Office 201, Jordi Girona 1-3, 08034 Barcelona, Spain). Corresponding author: X. Ros-Roca, xavier.ros.roca@upc.edu. 


\title{
Investigating the Quality of Spiess-Like and SPSA approaches for Dynamic OD Matrix Estimation
}

\begin{abstract}
Since OD matrices are not directly observable, indirect procedures have been developed to estimate OD matrices from traffic data. Traffic management practice must move toward dynamic traffic assignment models because they capture congestion propagation effects. In this context, dynamic OD matrices are needed. This paper first explores the extension of a well-known bilevel analytical static method, (Spiess 1990) to the dynamic context, then analyses the solutions obtained regarding both convergence to measured traffic data and structural similarity to an a priori OD matrix. A simulation optimization technique, SPSA, is then proposed because its flexibility allows inclusion of traffic counts (as in Spiess method) and emerging ICT traffic measurements. The performances of these two types of algorithms are analysed in detail, focussing on faults of classical convergence measures to obtain an estimated dynamic OD matrix structurally similarity to the a priori OD matrix and pros and cons of variants of selected methods.
\end{abstract}

Keywords: traffic simulation; OD estimation; simulation optimization; traffic assignment

\section{Introduction}

\subsection{Motivation and problem formulation}

Origin to Destination (OD) matrices that describe the mobility patterns across a road network serve as the main input to most traffic models, namely the dynamic traffic models implementing either Dynamic Traffic Assignment (DTA) or Dynamic User Equilibrium (DUE) approaches. The estimation of OD matrices still represents a research challenge, since OD matrices are not yet observable or at least not fully observable. In spite of the advances made in taking traffic measurements using Information and Communication Technologies (ICT), they only provide partial estimates which alone are not yet sufficient to provide unquestionable estimates and must be fused with data from other sources. These fusion processes pertain to research on better ways to adjust or correct the initial estimate of an existing OD matrix, $\boldsymbol{X}^{\boldsymbol{H}}$, which is usually called the 
"historical" or "target” OD matrix. These corrections are based on a set of traffic measurements, usually traffic volumes $\widehat{\boldsymbol{Y}}$, which are measured in a subset of links on the road network.

The conventional approaches are usually based on a bilevel optimization approach, generally considered to be the most efficient formulation for OD matrix adjustment exploiting traffic volumes $\widehat{\boldsymbol{Y}}$, that are measured in a subset of links of the road network. Its efficiency is based on its explicitly taking into account the congestion effects that influence the use of paths between OD pairs. The most common formulation considers the available sources of data to be:

- An historical or seed OD matrix $\boldsymbol{X}^{\boldsymbol{H}}$ that is usually made available by a household survey or a former demand model.

- Traffic volumes (i.e., link flow counts $\widehat{\boldsymbol{Y}}$ ) that are measured by detection stations (i.e., link count posts) for a subset of links $l \in \hat{L} \subseteq L$ in the network, where $L$ is the set of links.

The problem is then formulated as an optimization problem:

$$
\begin{gathered}
\min Z(\boldsymbol{X}, \boldsymbol{Y})=w_{1} F_{1}\left(\boldsymbol{X}, \boldsymbol{X}^{\boldsymbol{H}}\right)+w_{2} F_{2}(\boldsymbol{Y}, \widehat{\boldsymbol{Y}}) \\
\boldsymbol{Y}=A \operatorname{ssignmt}(\boldsymbol{X}) \\
\boldsymbol{X} \geq 0
\end{gathered}
$$

where $F_{1}$ and $F_{2}$ are suitable distance functions between estimated and observed values; while $w_{1}$ and $w_{2}$ are weighting factors reflecting the uncertainty of the information contained in $\boldsymbol{X}^{\boldsymbol{H}}$ and $\widehat{\boldsymbol{Y}}$, respectively. The underlying hypothesis is that $\boldsymbol{Y}(\boldsymbol{X})$ are the link flows predicted by 
assigning the demand matrix $\boldsymbol{X}$ onto the network, which can be expressed by a proportion of the OD demand flows passing through the count location at a certain link. In terms of the assignment matrix $\boldsymbol{A}(\boldsymbol{X})$, which is the proportion of OD flow that contributes to a certain link traffic count, is:

$$
Y=A(X) X
$$

This is a bilevel optimization problem that solves (at the upper level) the nonlinear optimization problem by substituting the estimated flows $\boldsymbol{Y}$ in the objective function of (1) with the relationship (2):

$$
\begin{gathered}
\min Z(\boldsymbol{X}, \boldsymbol{Y})=w_{1} F_{1}\left(\boldsymbol{X}, \boldsymbol{X}^{\boldsymbol{H}}\right)+w_{2} F_{2}(\boldsymbol{A}(\boldsymbol{X}) \boldsymbol{X}, \widehat{\boldsymbol{Y}}) \\
\boldsymbol{X} \geq 0
\end{gathered}
$$

To estimate a new assignment matrix $\boldsymbol{X}$, while at the lower level, a static user equilibrium assignment is used to solve the assignment problem $\boldsymbol{Y}=\operatorname{Assignmt}(\boldsymbol{X})$ in order to estimate the assignment matrix $\boldsymbol{A}(\boldsymbol{X})$ induced by the new $\boldsymbol{X}$. (Spiess 1990) is a good example of a seminal model based on this approach.

\subsection{Objectives}

The research reported in this paper has three main objectives. First, our goal is to explore extending the analytical approaches to the dynamic case. In other words, instead of a unique historical OD matrix $\boldsymbol{X}^{\boldsymbol{H}}$ for a unique time interval covering the whole-time horizon $T$ of the analysis, we use a set of historical OD matrices $\boldsymbol{X}_{\boldsymbol{r}}^{\boldsymbol{H}}$ for each of the time intervals $r$ in which the 
time horizon $T$ has been split. At the same time, these traffic counts are assumed to be available for each time interval $t$ in time horizon $T$. The link flow measurements $\left\{\hat{Y}_{l, t}\right\}$ are measured by detection stations at the subset of links $l \in \hat{L} \subseteq L$ in the network.

Second, a further goal is to explore extending the dynamic case of an alternative analytical approach for solving the bilevel model (1) through a simulation optimization approach based on using the Simultaneous Perturbation Stochastic Approximation (SPSA) (Spall 1992, 1998).

In both cases, if the dynamic approach accounts for the time dependency of the historical matrix $\boldsymbol{X}_{\boldsymbol{r}}^{H}$, then the static assignment must be replaced by a dynamic assignment in order to solve the lower level problem. The static assignment would not be able to account for either the time evolution of the congestion or the consequent rerouting; therefore, there would remain no accounting for the changes in the assignment matrix that determines link flows. However, emulating these behaviours is exactly what is expected from a dynamic assignment. Replacing the static assignment with the dynamic one requires a reformulation of the usual approaches. Analysing this reformulation and its computational performance is one objective of this paper. The dynamic assignment engine used in our case is the Simulation-based Assignment (SBA) implemented in Visum-18 (PTV AG 2018).

Our third objective addresses an additional question that needs further research, namely in regard to measuring the quality of the corrected OD matrix with the adjustment procedure. This is usually handled by the distance matrices $F_{1}$ and $F_{2}$ in the objective function in (1). Assuming that, if $F_{1}$ and $F_{2}$ are distance functions, then the minimization process will achieve the expected results, which are presumably confirmed a posteriori by the regression analysis of the observed $\widehat{\boldsymbol{Y}}$ and estimated $Y$ link flow counts. However, given that the problem is highly 
underdetermined, there could exist different solutions that provide the same values for the conventional distance functions $F_{1}$ while having different structures (Djukic 2014) and similar values of $R^{2}$. In that case, it does not seem that the correlation coefficient is a good indicator of the quality; rather, it simply shows that the estimated $\boldsymbol{Y}$ link flow counts, calculated by (2) from the corrected matrix $\boldsymbol{X}$, are close enough to the observed $\widehat{\boldsymbol{Y}}$ link flows. However, it may happen that the model (1) acts as a meta-regression model by pulling or pushing the OD trips $\boldsymbol{X}$ just to fit link flows in terms of the paths using the links, independently of the underlying spatial-temporal structure of the OD demand. Thus, to state it more explicitly: the third objective of this paper is to analyse the structural similarity of the estimated OD matrix with respect to the a priori OD matrix.

\section{Analytical Formulations}

Static models have made wide use of the analytical approaches that include flow counts as complementary information to-reduce underdetermination when solving the described minimization problem (1) (Codina and Montero 2006; Lundgren and Peterson 2008; Spiess 1990). The reason for this is because they are algorithmically efficient and present nice properties for convergence and stability. However, since they are static, they are supported by static assignment models.

Some researchers (Frederix, et al. 2013; Lundgren and Peterson 2008; Toledo and Kolechkina 2013; Yang et al.2017) drew attention to the role played by the quality of the assignment matrix, which results from the lower level assignment process when estimating the flows used in the upper level. Therefore, those researchers have proposed either analytical or empirical approaches for improving it. The analytical approaches assume a functional 
dependency that allows for a Taylor expansion around the current solution. While some authors (Lundgren and Peterson 2008) still derive the expansion from a static traffic assignment, others (Frederix et al. 2013; Toledo and Kolechkina 2013) propose a dynamic traffic assignment to account for time dependencies. This is appropriate for working with congestion building processes that would be captured by the analytical expansion of the assignment matrix. While (Frederix et al. 2013) offers a relevant theoretical contribution, (Toledo and Kolechkina 2013) provides more insight into applying it in larger networks- However, their paper mentions Spiess as one of the tested alternatives, and yet they do not provide further details about its extension to dynamic scenarios. It seems to be a particular case of their approach, which uses second-order derivatives and complex numerical optimization procedures (e.g., Armijo rules to compute the step length), which require higher computational effort. Therefore, this paper seeks to validate the modification of the Spiess procedure using, on the one hand, a first-order approach to the assignment matrix that is provided by a Dynamic Traffic Assignment; and, on the other, an ad hoc reformulation of the analytical calculation of the gradient that is suitable for a straightforward calculation of the step length at each iteration.

Throughout this paper, the following notation is used:

- $I$ is the set of OD pairs.

- $\mathcal{T}=\{1, \ldots, T\}$ is the set of time intervals.

- $L$ is the set of links in the network. $\hat{L} \subseteq L$ is the subset of links that have sensors. $\hat{y}_{l, t}$ are the measured flow counts at link $l$ during time period $t . y_{l, t}$ are the corresponding simulated flow counts, $\forall l \in \hat{L} \subseteq L$ and $\forall t \in \mathcal{T} . \boldsymbol{Y}=\left(y_{l, t}\right)$ and $\widehat{\boldsymbol{Y}}=\left(\hat{y}_{l, t}\right)$ are the link flow counts in vector form. 
- $x_{n, r}$ are the OD flows for $n$-th OD pairs departing during time period $r, \forall n \in I$ and $\forall r \in$ $\mathcal{T} . \boldsymbol{X}=\left(x_{n, r}\right)$ are the OD flows in vector form.

- $a_{n, r}^{l, t}$ is the flow proportion of the $n$-th OD pair, $n \in I$, departing at time period $r \in \mathcal{T}$ and captured by link $l \in \hat{L}$ at time period $t \in \mathcal{T} . \boldsymbol{A}=\left[a_{n, r}^{l, t}\right]$ is the assignment matrix.

Given a network with a set of links $L$, a set $I$ of OD pairs, and the set of time periods $\mathcal{T}$. The goal of the dynamic OD-matrix estimation problem is to find a feasible vector (OD-matrix) $\boldsymbol{X}^{*} \in \mathrm{G} \subseteq \mathbb{R}_{+}^{I \times \mathcal{T}}$, where $\boldsymbol{X}^{*}=\left(x_{n, r}^{*}\right), n \in I, r \in \mathcal{T}$, consists of the demands for all OD pairs. It can be assumed that, when assigning the time-sliced OD matrices onto the links of the network, it should be done according to an assignment proportion matrix $\boldsymbol{A}=\left[a_{n, r}^{l, t}\right], \forall l \in L, \forall n \in$ $I, \forall r, t \in \mathcal{T}$, where each element in the matrix is defined as the proportion of the OD demand $x_{n, r}$ that uses link $l$ at time period $t$. The notation $\boldsymbol{A}=\boldsymbol{A}(\boldsymbol{X})$ is used to indicate that, in general, these proportions depend on the demand. The linear relationship between the flow count on a link and the given OD pair is in matrix form, which thus sets the vector of detected flows as $\boldsymbol{Y}=$ $\left(\boldsymbol{Y}_{1}, \ldots, \boldsymbol{Y}_{\boldsymbol{T}}\right)=\left(y_{1,1}, \ldots, y_{L, 1}, \ldots, y_{1, T}, \ldots y_{L, T}\right)$ and the vector of OD flows as $\boldsymbol{X}=\left(\boldsymbol{X}_{\mathbf{1}}, \ldots, \boldsymbol{X}_{\boldsymbol{T}}\right)=$ $\left(x_{1,1}, \ldots, x_{N, 1}, \ldots, x_{1, T}, \ldots, x_{N, T}\right)$. The relationship can be expressed as a matrix product, that is:

$$
\begin{gathered}
\boldsymbol{Y}=\boldsymbol{A}(\boldsymbol{X}) \cdot \boldsymbol{X} \\
\text { with } \boldsymbol{A}(\boldsymbol{X})=\left(\begin{array}{cccc}
\boldsymbol{A}^{\mathbf{1 , 1}} & \mathbf{0} & \cdots & \mathbf{0} \\
\boldsymbol{A}^{\mathbf{1 , 2}} & \boldsymbol{A}^{\mathbf{2 , 2}} & \mathbf{0} & \vdots \\
\vdots & \ddots & \ddots & \mathbf{0} \\
\boldsymbol{A}^{\mathbf{1 , T}} & \cdots & \boldsymbol{A}^{T-\mathbf{1 , T}} & \boldsymbol{A}^{\boldsymbol{T , T}}
\end{array}\right) \text { where } \boldsymbol{A}^{r, \boldsymbol{t}}=\left(\begin{array}{ccc}
a_{1, r}^{1, t} & \cdots & a_{N, r}^{1, t} \\
\vdots & \ddots & \vdots \\
a_{1, r}^{L, t} & \cdots & a_{N, r}^{L, t}
\end{array}\right)
\end{gathered}
$$

where $a_{n, r}^{l, t}$ represents the proportion of OD flow departing at time $r, x_{n, r}$, passing through link $l$ at time $t, y_{l, t} . \boldsymbol{A}^{r, t}$ represents the assignment matrix for the departing flows at time window $r$ 
detected at time window $t$ and, therefore, $\boldsymbol{A}$ is a lower-diagonal matrix, because OD flow departing at time $r$ cannot pass through link $l$ at time $t<r$.

This linear mapping between the link flows and the OD flows is indeed the first term in the Taylor expansion of the relationship between link flows and OD flows, where additional terms capture the assignment matrix's sensitivity to changes in the OD flows, path choice and congestion propagation effects (Frederix et al. 2011, 2013; Toledo and Kolechkina 2013). Let $\boldsymbol{X}^{\prime}$ be in the neighbourhood of $\boldsymbol{X}$. Then, the Taylor expansion is:

$$
\begin{aligned}
y_{l, t} & =\sum_{n \in I} \sum_{r=1}^{t} a_{n, r}^{l, t}\left(\boldsymbol{X}^{\prime}\right) x_{n, r}^{\prime}+\sum_{n \in I} \sum_{r=1}^{t} \frac{\partial y_{l, t}\left(\boldsymbol{X}^{\prime}\right)}{\partial x_{n, r}}\left(x_{n, r}-x_{n, r}^{\prime}\right)= \\
& =\sum_{n \in I} \sum_{r=1}^{t} a_{n, r}^{l, t}\left(\boldsymbol{X}^{\prime}\right) x_{n, r}^{\prime}+\left.\sum_{n \in I} \sum_{r=1}^{t} \frac{\partial\left[\sum_{n \in I} \sum_{r=1}^{t} a_{n, r}^{l, t}\left(\boldsymbol{X}^{\prime}\right) x_{n, r}\right]}{\partial x_{n, r}}\right|_{\boldsymbol{X}^{\prime}}\left(x_{n, r}-x_{n, r}^{\prime}\right)= \\
& =\sum_{n \in I} \sum_{r=1}^{t} a_{n, r}^{l, t}\left(\boldsymbol{X}^{\prime}\right) x_{n, r}^{\prime}+\sum_{n \in I} \sum_{r=1}^{t}\left(x_{n, r}-x_{n, r}^{\prime}\right)\left[\left.\sum_{n^{\prime} \in I} \sum_{r^{\prime}=1}^{t} \frac{\partial a_{n^{\prime}, r^{\prime}}^{l, t}\left(\boldsymbol{X}^{\prime}\right)}{\partial x_{n, r}}\right|_{\boldsymbol{X}^{\prime}} x_{n^{\prime}, r^{\prime}}^{\prime}\right]
\end{aligned}
$$

Since one of the objectives of our research is to investigate extending static cases to dynamic cases and, given that Spiess’ static approach (Spiess 1990) is one of the most robust in practice, we decided to redefine Spiess’ approach to the dynamic case by simply using the first term in the above Taylor expansion. It does not account for the propagation effects, but it explicitly considers the time dependencies.

\subsection{Dynamic Spiess algorithm}

The extrapolation of the Spiess method to a dynamic traffic assignment (DTA) is possible by including the time windows to the entire formulation, as follows: 


$$
\begin{gathered}
\min Z(\boldsymbol{X})=\frac{1}{2} \sum_{t \in \mathcal{T}} \sum_{l \in \hat{L}}\left(\left(\sum_{n \in I} \sum_{r=1}^{t} a_{n, r}^{l, t} x_{n, r}\right)-\hat{y}_{l, t}\right)^{2} \\
a_{n, r}^{l, t}=\operatorname{Assignment}(\boldsymbol{X}) \\
x_{n, r} \geq 0
\end{gathered}
$$

where $a_{n, r}^{l, t}$ is the assignment matrix described before. Therefore, the linear combination inside the brackets is the simulated flow $y_{l, t}$, and

$$
\frac{\partial y_{l, t}}{\partial x_{n, r}}=a_{n, r}^{l, t}
$$

As in (Spiess 1990), the chain rule can be used to obtain the gradient of the objective function:

$$
\frac{\partial Z}{\partial x_{n, r}}=\sum_{t \in \mathcal{T}} \sum_{l \in \hat{L}} \frac{\partial y_{l, t}}{\partial x_{n, r}}\left(y_{l, t}-\hat{y}_{l, t}\right)=\sum_{t \in \mathcal{T}} \sum_{l \in \hat{L}} a_{n, r}^{l, t}\left(y_{l, t}-\hat{y}_{l, t}\right)
$$

And to find the optimal step size by using the same procedure, we obtain similar equations:

$$
y_{l, t}^{\prime}=\frac{d y_{l, t}}{d \lambda}=\sum_{r=1}^{t} \sum_{n \in I} \frac{d x_{n, r}}{d \lambda} \frac{\partial y_{l, t}}{\partial x_{n, r}}=\sum_{r=1}^{t} \sum_{n \in I}-x_{n, r} \frac{\partial Z}{\partial x_{n, r}} \frac{\partial y_{l, t}}{\partial x_{n, r}}
$$

The optimal step length $\lambda$ can then be calculated solving a 1-dimensional optimization problem, whose solution is given by: 


$$
\begin{gathered}
\mathrm{Z}^{\prime}(\lambda)=\sum_{t \in \mathcal{T}} \sum_{l \in \hat{L}} y_{l, t}^{\prime}\left(\tilde{y}_{l, t}-\hat{y}_{l, t}+\lambda y_{l, t}^{\prime}\right)=0 \\
\lambda^{*}=\frac{-\sum_{t \in \mathcal{T}} \sum_{l \in \hat{L}} y_{l, t}^{\prime}\left(y_{l, t}-\hat{y}_{l, t}\right)}{\sum_{t \in \mathcal{T}} \sum_{l \in \hat{L}} y_{l, t}^{\prime 2}}
\end{gathered}
$$

And then, the iterative procedure described by (Spiess 1990) can be used in DTA using these new equations, which become expanded with the time windows.

\subsection{Dynamic Spiess variants}

Variants of Dynamic Spiess have been tested, aimed at improving computational performance by decreasing the number of assignments, or adding a distance term between OD matrices in the Objective Function.

\subsubsection{Reassigning at convergence}

As in (Spiess 1990), the iterative procedure used to solve the minimization problem (6) employs gradient methods (8) and (10), and this requires one full assignment of the OD matrices at each single iteration of the minimization procedure. The assignment matrix, $a_{n, r}^{l, t}$, depends directly on $\boldsymbol{X}=\left(x_{n, r}\right)$; so each iteration of the minimization problem requires a single assignment of $\boldsymbol{X}$ onto the network, which increases the computational time. Let us assume that $a_{n, r}^{l, t}$ does not change significantly at each iteration. Then, reassigning at every single iteration is not needed and the computational time is therefore significantly reduced, because DTA assignment is a time-consuming procedure. In this case, the iterations must be distinguished between Major iterations (when an assignment is required at the lower level) and Minor iterations (when only the minimization iterations at the upper level are considered). 
The jump from the upper level to the lower level is made only when a convergence criterion is satisfied. The assignment matrix is then recalculated, and more minor iterations are launched.

\subsubsection{Quadratic function for $\boldsymbol{X}^{\boldsymbol{H}}$}

Furthermore, we have added a second term in the objective function in order to compare it to a historical OD matrix. In a first approach, the quadratic function is used:

$$
\min Z=\frac{1}{2} \sum_{t \in \mathcal{T}} \sum_{l \in \hat{L}}\left(\left(\sum_{n \in I} \sum_{r=1}^{t} a_{n, r}^{l, t} x_{n, r}\right)-\hat{y}_{l, t}\right)^{2}+\frac{w}{2} \sum_{r \in \mathcal{T}} \sum_{n \in I}\left(x_{n, r}-x_{n, r}^{H}\right)^{2}
$$

In this case, Equation (8) is updated as:

$$
\frac{\partial Z}{\partial x_{n, r}}=\sum_{t \in \mathcal{T}} \sum_{l \in \hat{L}} \frac{\partial y_{l, t}}{\partial x_{n, r}}\left(y_{l, t}-\hat{y}_{l, t}\right)+\frac{w}{2} x_{n, r}=\sum_{t \in \mathcal{T}} \sum_{l \in \hat{L}} a_{n, r}^{l, t}\left(y_{l, t}-\hat{y}_{l, t}\right)+\frac{w}{2} x_{n, r}
$$

\subsubsection{Entropy function for $\boldsymbol{X}^{\boldsymbol{H}}$}

Due to criticism of using Euclidean distances (Frederix et al. 2013), using a different distance function is suggested. Furthermore, (Djukic 2014) also shows that using a Euclidian distance term can result in two matrices that have very different structures but maintain the same distance value with respect to the reference matrix. Although additional measurements are expected to improve the outcome of the OD estimation in terms of structural similarity, the analytic approaches do not seem capable to add measurements that are different from the link counts.

The classical entropy function has been chosen because of its structural meaning. Then, equations (11) and (12) become: 


$$
\begin{aligned}
\min Z & =\frac{1}{2} \sum_{t \in \mathcal{T}} \sum_{l \in \hat{L}}\left(\left(\sum_{n \in I} \sum_{r=1}^{t} a_{n, r}^{l, t} x_{n, r}\right)-\hat{y}_{l, t}\right)^{2}+\frac{w}{2} \sum_{r \in \mathcal{T}} \sum_{n \in I} x_{n, r} \cdot \log \left(\frac{x_{n, r}}{x_{n, r}^{H}}\right) \\
\frac{\partial Z}{\partial x_{n, r}} & =\sum_{t \in \mathcal{T}} \sum_{l \in \hat{L}} a_{n, r}^{l, t}\left(y_{l, t}-\hat{y}_{l, t}\right)+\frac{w}{2}\left(\log \left(\frac{x_{n, r}}{x_{n, r}^{H}}\right)+1\right)
\end{aligned}
$$

\section{Simulation Optimization Techniques: Simultaneous Perturbation Stochastic Approximation (SPSA)}

The optimization problem (1), as mentioned already, is highly underdetermined because there are many more variables than equations in the system. In other words, $\boldsymbol{X} \in \mathbb{R}^{|I| \times T}, \boldsymbol{Y} \in \mathbb{R}^{|\hat{L}| \times T}$ and $|I| \gg|\widehat{L}|$, and therefore the problem is very sensitive to the quantity of data and the detection layout in the real network. Thus, because the availability of new measurements like those provided by Smartphone and GPS localization allows calculating travel times between arbitrary pairs of points, this seems to be a promising approach for reducing this underdetermination. As mentioned before, it is unclear how these new measurements can be included in the analytical formulations, but it seems rather easy to deal with them by using approaches based on derivativefree optimization methods that approximate the descent direction based on simulation. Among them, simulation optimization techniques are especially suited to optimization problems that cannot be solved with the usual analytical algorithms due to reasons such as:

- The objective function cannot be analytically expressed as a function of parameters because its evaluation requires a simulation. Therefore, it is not differentiable in terms of the parameters.

- The time cost of evaluating the objective function is expensive in regard to having simulated data for each evaluation of the function. 
There is a wide range of different simulation optimization techniques, such as NelderMead, SNOBFIT and SPSA. (Osorio and Chong 2015) makes an approximation of the upper level function by building a metamodel that can be solved analytically. SPSA preserves the original upper level formulation and is easy to implement for simulation optimization problems.

Simultaneous Perturbation Stochastic Approximation (SPSA) is commonly used in OD matrix estimation (Antoniou et al. 2015; Cantelmo et al. 2014; Cipriani et al. 2011; Lu et al. 2015), and it can easily account for additional measurements (Antoniou et al. 2016; Bullejos et al. 2014; Carrese et al. 2017; Nigro et al. 2018). However, before extending its use, we have considered it necessary to learn about its computational performance.

SPSA (Spall 1992) is a simulation-based optimization algorithm, and it requires only two evaluations of the objective function for approximating the gradient instead of $N$, as in the case of a finite-difference gradient approach. As in many iterative procedures, it begins with an initial OD matrix (usually a historical OD matrix), and the next point is computed using the first order Taylor development:

$$
\boldsymbol{X}_{k+1}=\boldsymbol{X}_{k}-a_{k} \widehat{\boldsymbol{g}}_{\boldsymbol{k}}\left(\boldsymbol{X}_{k}\right)
$$

Two particularities distinguish this method from the gradient descent method:

- The estimated gradient $\widehat{\boldsymbol{g}}_{k}\left(\boldsymbol{X}_{k}\right)$, is calculated as:

$$
\widehat{\boldsymbol{g}}_{k}\left(\boldsymbol{X}_{k}\right)=\frac{Z\left(\boldsymbol{X}_{k}+c_{k} \boldsymbol{\Delta}_{k}\right)-Z\left(\boldsymbol{X}_{k}\right)}{c_{k}} \cdot\left(\begin{array}{c}
\Delta_{k, 1}^{-1} \\
\vdots \\
\Delta_{k, N}^{-1}
\end{array}\right)=\left(\begin{array}{c}
\frac{Z\left(\boldsymbol{X}_{k}+c_{k} \boldsymbol{\Delta}_{k}\right)-Z\left(\boldsymbol{X}_{k}\right)}{c_{k} \Delta_{\mathrm{k}, 1}} \\
\vdots \\
\frac{Z\left(\boldsymbol{X}_{k}+c_{k} \boldsymbol{\Delta}_{k}\right)-Z\left(\boldsymbol{X}_{k}\right)}{c_{k} \Delta_{\mathrm{k}, \mathrm{N}}}
\end{array}\right)
$$


where $\Delta_{\mathbf{k}}$ is a random perturbation $\mathrm{N}$-dimensional vector with $\Delta_{i}, \forall i$ independent identically distributed random variables that satisfy $\mathbb{E}\left(\Delta_{i}\right)=0$ and $\left|\mathbb{E}\left(\left(\Delta_{i}^{-1}\right)^{n}\right)\right|<\infty, \forall n$. One commonly used perturbation is $\Delta_{i} \sim B e(1 / 2, \pm 1)$, which is a Bernoulli distribution with a probability of $1 / 2$ for each \pm 1 . This is the asymmetric design, although a symmetric design using $Z\left(\boldsymbol{X}_{k}+c_{k} \Delta_{k}\right)$ and $Z\left(\boldsymbol{X}_{k}-c_{k} \Delta_{k}\right)$ can also be considered.

- The spacing coefficient $c_{k}$ and the step size $a_{k}$ are decreasing sequences of positive real values, and they satisfy some regularity conditions in order to ensure the convergence of the method, as detailed in (Spall 1992). Typically, the sequences used are:

$$
a_{k}=\frac{a}{(A+k+1)^{\alpha}}, \quad c_{k}=\frac{c}{(k+1)^{\gamma}}
$$

where $a, A$ and $c$ are fixed and depend on the problem, while $\alpha=0.602$ and $\gamma=0.101$.

In (Spall 1992), it is shown that averaging many independent estimates of the gradient of Equation (15) contributes to a more stable and quicker convergence of the SPSA method. Therefore, the gradient estimation is finally calculated as:

$$
\widehat{\boldsymbol{g}}\left(\boldsymbol{X}_{\boldsymbol{k}}\right)=\frac{1}{n_{g}} \sum_{j=1}^{n_{g}} \widehat{\boldsymbol{g}}_{\boldsymbol{k}}^{j}\left(\boldsymbol{X}_{\boldsymbol{k}}\right)
$$

where $\widehat{\boldsymbol{g}}_{\boldsymbol{k}}^{j}\left(\boldsymbol{X}_{\boldsymbol{k}}\right)$ is calculated as in Equation (15). The asymmetric design for the gradient saves a large number of assignments, since all $\widehat{\boldsymbol{g}}_{\boldsymbol{k}}^{j}\left(\boldsymbol{X}_{\boldsymbol{k}}\right), \forall j$ share the mid-point $\boldsymbol{X}_{\boldsymbol{k}}$ evaluation.

\subsection{SPSA variants}

The versatility of simulation optimization techniques - especially when using SPSA - allows us 
to include additional information in a newer form, such as the constraints in the OD estimation problem. (Ros-Roca et al. 2017) tried adding constraints to simulation optimization problems when dealing with the calibration of microsimulation models. In this case, we have tested variants of the method and different innovative techniques (explained below).

\subsubsection{Conjugate Gradient (CG) Descent version}

Some researchers in past works (Bullejos et al. 2014; Cantelmo et al. 2014) used the Conjugate Gradient (CG) (Luenberger and Ye 1973) descent method for the optimization procedures of the OD estimation problem. This modifies the descent direction in the iterative procedure by using the previous iteration gradient. It can be incorporated into SPSA by replacing Equation (14) with:

$$
\begin{gathered}
\boldsymbol{X}_{k}=\boldsymbol{X}_{k-1}+a_{k} \boldsymbol{d}_{k} \\
\boldsymbol{d}_{\boldsymbol{k}}=-\widehat{\boldsymbol{g}}\left(\boldsymbol{X}_{\boldsymbol{k}}\right)+\beta_{k} \widehat{\boldsymbol{g}}\left(\boldsymbol{X}_{k-1}\right) \quad, \quad \beta_{k}=\frac{\widehat{g}\left(\boldsymbol{X}_{k}\right)^{T} \boldsymbol{d}_{k-1}}{\left\|\boldsymbol{d}_{k-1}\right\|^{2}}
\end{gathered}
$$

\subsubsection{Normalization of variables}

SPSA's main drawback for the OD estimation problem is that all different OD flows receive the same perturbation magnitude, Equation (14). Furthermore, OD flows usually have very different magnitudes, which imply very different changes to each flow, and this can lead to several problems of convergence. (Tympakianaki et al. 2015) approached this phenomenon by clustering the variables according to their magnitudes. This work does so by normalizing to the interval $[0,1]$ all variables using some reasonable bounds for each variable, $\left[a_{i}, b_{i}\right]$ and a classical linear transformation from $\left[a_{i}, b_{i}\right]$ to $[0,1]$, where $a_{i}$ and $b_{i}$ are the reasonable lower and upper bounds 
based on additional information from the network, such as socioeconomic or past reliable OD matrices, as in (Ros-Roca et al. 2018). Normalization is performed using the linear application:

$$
\begin{aligned}
\varphi_{i}: \quad\left[a_{i}, b_{i}\right] & \rightarrow \quad[0,1] \\
X_{i} & \mapsto \quad \frac{X_{i}-a_{i}}{b_{i}-a_{i}}
\end{aligned}
$$

Using the normalized variables in SPSA procedure, each variable will be perturbed according to its magnitude.

\subsubsection{Selection of SPSA Gain Sequences}

Experience with similar problems shows that the selection of SPSA gain sequences, $a_{k}, c_{k}$, is crucial for the convergence and performance of the algorithm. The sequences in the form of Equation (16) are widely used, as they satisfy the conditions of convergence that were proved in (Spall 1992). This reduces the problem to selecting appropriate values for $a, A, \alpha, c$ and $\gamma$. (Kostic et al. 2017) show the sensitivity of SPSA with respect to these parameters, but no research has been conducted on finding a criterion to select them in an automatized procedure. Based on the guidelines in (Spall 2003), an automated selection of the parameters $a, A$ and $c$ is proposed in the current work and it has been implemented. The choice of these parameters is based on the objective function's variability that results from the simulation and the desired perturbation steps in the early iterations. The schema is detailed below:

(1) Fix $\alpha=0.602, \gamma=0.101$ as (Spall 1998) determines, where it is stated that they are optimal values for the convergence. 
(2) Compute several evaluations of $Z\left(\boldsymbol{X}^{H}\right)$ to capture the variability of the objective function. Since the variables have been normalized, it seems natural to use the coefficient of variation $\left(\operatorname{CoV}(Z)=\sigma_{Z} / \mu_{Z}\right)$. The parameter $c$ is set at $c=\operatorname{CoV}$.

(3) Set $A$ as $10 \%$ of the maximum number of iterations $\left(A=0.1 \cdot\right.$ iter $\left._{\max }\right)$.

(4) Simulate $n_{g}$ experiments using the SPSA logic $\boldsymbol{X}_{i}=\boldsymbol{X}^{H}+c \boldsymbol{\Delta}_{N}$ and find the respective gradients, $\boldsymbol{g}_{k}$, as in the SPSA procedure.

(5) Determine the desired iterative modification of the first iteration:

$$
\boldsymbol{X}_{k+1}=\boldsymbol{X}_{k}-a_{k} \boldsymbol{g}_{k} \rightarrow \boldsymbol{X}_{k+1}-\boldsymbol{X}_{k}=\left|a_{k} \boldsymbol{g}_{k}\right|
$$

(6) Therefore, one can compute the corresponding $a$ for the desired change for the initial iteration:

$$
\left|a_{k} \boldsymbol{g}_{k}\right|=\frac{a}{(1+A+k)^{\alpha}}\left|\boldsymbol{g}_{k}\right| \rightarrow a=\frac{\left|a_{k} \boldsymbol{g}_{k}\right|(1+A+k)^{\alpha}}{\left|\boldsymbol{g}_{k}\right|}
$$

Since $n_{g}$ experiments have been performed, one has to choose the minimum, which is calculated as above, that is:

$$
a=\min \left\{a_{\{i=1\}}, \ldots, a_{\left\{i=N_{g}\right\}}\right\}
$$

\subsubsection{SPSA Variants: Constrained SPSA and Penalized SPSA}

As already mentioned, the underdetermination of the OD estimation problem can lead to different adjusted OD matrices that show the same traffic counts at the sensor locations even though they are different. Furthermore, the adjusted OD matrix can also be non-consistent with 
the socioeconomic factors of the area under study. In traffic studies, practitioners usually have access to historical data in the form of an OD matrix, $\boldsymbol{X}^{\boldsymbol{H}}$, which, with a certain degree of uncertainty, provides prior information about the mobility patterns of the study area. Therefore, including constraints in the SPSA formulation that accounts for this information could provide more realistic results. Our first approach was to add bounding values to the OD values, which is not easy to do in analytical formulations (Codina and Montero 2006) but is relatively easy to manage in SPSA. In (Cipriani et al. 2011), a single generation constraint is added to the minimization problem:

$$
\sum_{i=1}^{n_{h}} G_{o}^{i} \leq G_{o}^{*}, \forall o \in\{\text { origins }\}
$$

with $G_{o}^{*}$ being the a priori generation value for the origin zone $o$ and $n_{h}$ the number of time periods. The proposal in this paper specifies upper and lower bounds for each OD flow. This can be a percentage of the historical value $\beta$, which is in accordance with its degree of uncertainty. The minimization problem is then updated with the constraints:

$$
\begin{aligned}
& \min Z(\boldsymbol{X}, \boldsymbol{Y})=w_{1} F_{1}\left(\boldsymbol{X}, \boldsymbol{X}^{\boldsymbol{H}}\right)+w_{2} F_{2}(\boldsymbol{Y}, \widehat{\boldsymbol{Y}}) \\
& \boldsymbol{Y}=\text { Assignmt }(\boldsymbol{X}) \\
& \boldsymbol{X} \in G=\left\{(1-\beta) x_{n, r}^{H} \leq x_{n, r} \leq(1+\beta) x_{n, r}^{H}, \forall x_{n, r} \in \boldsymbol{X}\right\} \subset \mathbb{R}_{+}^{I \times T} \\
& \boldsymbol{X} \geq 0
\end{aligned}
$$

This single constraint in (24) is the constraint that results from summing all the upper bounds in (23) for each origin. The summation of all the constraints makes the feasible region bigger, thus permitting greater values among some variables, which is compensated by other low 
values among others. This proposal for constrained SPSA defines a smaller feasible region that accounts for further information for each OD pair.

The constraints that have been added to the problem also have an effect on the originally presented SPSA algorithm. (Sadegh and Spall 1998) proposed adding a projection to the set $G$ during the iterative procedure shown in Equation (14). The projection is applied to only the iterative procedure as $\boldsymbol{X}_{\boldsymbol{k}+\mathbf{1}}=\pi_{G}\left(\boldsymbol{X}_{\boldsymbol{k}}-a_{k} \widehat{\boldsymbol{g}}_{\boldsymbol{k}}\left(\boldsymbol{X}_{\boldsymbol{k}}\right)\right)$, while $Z\left(\boldsymbol{X}_{k}+c_{k} \Delta_{k}\right)$ can be computed subject to non-negative OD values. This method, in which some strict constraints are added to the procedure, will be called Constrained SPSA.

Other formulation, inspired in (Wang and Spall 1999) equivalent to (24) based on adds penalty functions to the objective function, that is:

$$
\begin{gathered}
\min Z(\boldsymbol{X}, \boldsymbol{Y})=w_{1} F_{1}\left(\boldsymbol{X}, \boldsymbol{X}^{\boldsymbol{H}}\right)+w_{2} F_{2}(\boldsymbol{Y}, \widehat{\boldsymbol{Y}})+r_{k} P\left(\boldsymbol{X}, \boldsymbol{X}^{\boldsymbol{H}}\right) \\
\boldsymbol{Y}=\operatorname{Assignmt}(\boldsymbol{X}) \\
\boldsymbol{X} \geq 0
\end{gathered}
$$

where $r_{k}$ is an increasing sequence of the form $r_{k}=r(1+k)^{\rho}$, and $P\left(\boldsymbol{X}, \boldsymbol{X}^{\boldsymbol{H}}\right)$ is a set of penalization functions for the set of constraints that delimit the constraints of set $G$, formally:

$$
\begin{aligned}
G & \triangleq\left\{q_{n, r}\left(\boldsymbol{X}, \boldsymbol{X}^{\boldsymbol{H}}\right) \leq 0, \forall n \in I, r \in T\right\}= \\
& =\left\{x_{n, r}-(1+\beta) x_{n, r}^{H} \leq 0,(1+\beta) x_{n, r}^{H}-x_{n, r} \leq 0, \forall n \in I, r \in T\right\}
\end{aligned}
$$

The penalty function $P\left(\boldsymbol{X}, \boldsymbol{X}^{\boldsymbol{H}}\right)$ has to be differentiable, non-negative and an increasing function. (Wang and Spall 1999) propose a sum for each constraint of penalizing functions that satisfy $p(x)=0$ if and only if $x \geq 0$, such as 


$$
P\left(\boldsymbol{X}, \boldsymbol{X}^{\boldsymbol{H}}\right)=\sum_{n \in I} \sum_{r=1}^{T} w_{n, r} p\left(q_{n, r}\left(\boldsymbol{X}, \boldsymbol{X}^{\boldsymbol{H}}\right)\right)=\sum_{n \in I} \sum_{r=1}^{T} w_{n, r} \max \left\{0, q_{n, r}\left(\boldsymbol{X}, \boldsymbol{X}^{\boldsymbol{H}}\right)\right\}^{2}
$$

As in the previous variant, the iterative procedure is also modified to incorporate the gradient of the penalization function:

$$
\boldsymbol{X}_{k+1}=\boldsymbol{X}_{k}-a_{k} \widehat{\boldsymbol{g}}_{\boldsymbol{k}}\left(\boldsymbol{X}_{k}\right)-a_{k} r_{k} \boldsymbol{\nabla} \boldsymbol{P}\left(\boldsymbol{X}_{\boldsymbol{k}}, \boldsymbol{X}^{\boldsymbol{H}}\right)
$$

\section{Experimental framework}

The experimental framework used is of a synthetic nature, namely in that it selects a test network and assumes:

- The a priori knowledge of a Ground Truth (GT) OD matrix that represents the "real demand" (it will be noted as $\boldsymbol{X}^{\boldsymbol{G T}}$ along the article).

- A heuristic detection layout identifying the location of counting stations in the network.

- An SBA assignment of the Ground Truth Matrix. The estimated link flows resulting from the assignment at the links where the counting stations are located are taken as the "real measurements” in the computational experiments.

The objective of the computational experiments is to analyse the quality of the results, which are the measurements of the various performance indicators of the corrected OD matrices that are estimated by the methods analysed in this research work.

\subsection{Design Factors}

The design factors in the computational experiments for the selected network are: 
(1) Initializations of the target OD matrix. 6 different initializations have been used in order to contemplate different situations, from similar-structure matrices with different number of trips to non-similar-structure matrices:

- Incremental+: Incrementing all the OD values of the Ground Truth matrix by a fixed percentage: $\boldsymbol{I n c}^{+}=\boldsymbol{X}^{\boldsymbol{G T}}(1+\delta), \delta=0.25$.

- Incremental-: Decrementing all the OD values of the Ground Truth matrix by a fixed percentage: $\boldsymbol{I n \boldsymbol { c } ^ { - }}=\boldsymbol{X}^{\boldsymbol{G T}}(1-\delta), \delta=0.25$.

- Chaos: Equidistributing all the OD values of the Ground Truth matrix by rows while fixing generated trips by rows.

- Chaos+Inc+: Equidistributing all the OD values of the Ground Truth matrix by rows and incrementing all of them by the same $\delta=0.25$ fixed proportion.

- Chaos+Inc-: Equidistributing all the OD values of the Ground Truth matrix by rows and decrementing all of them by the same $\delta=0.25$ fixed proportion.

- Multitude: Adapted from (Antoniou et al. 2016) low demand initialization: $\boldsymbol{X}^{\boldsymbol{G T}}(r+q$. $\varepsilon)$, with $r=0.75, q=0.15$ and $\varepsilon \sim N(0,1 / 3)$.

Note that the Incremental+ and Incremental- OD matrices have the same spatial distribution structure or mobility pattern as the Ground Truth OD matrix because only a regular increment or decrement has been applied, while the Chaos matrices present a complete perturbation that changes the structure of the OD matrix. In other words, the mobility patterns between the Ground Truth and the historical OD matrix, used as the seed for the estimation procedure, are different. Figure 1(a) visualizes the differences between three of those OD matrices using heatmaps in a red-orange-yellow-white 
sequence, with red for null values and increasing to orange to yellow and to white as the flows increase. Regarding the total number of trips, the Ground Truth and the Chaos initializations have the same number of trips; the incremented OD matrices have $100 \delta \%$ more; and the decremented ones have $100 \delta \%$ less. The Multitude initialization aims to align with the Multitude benchmarking proposal.

(2) OD Adjustment methods. Two approaches and their variants have been considered in this case: first, the analytical approach discussed in Section 2; and second, the simulation optimization techniques described in Section 3.

\subsection{Test site}

A model of the city of Hillsboro, USA, has been selected for these experiments. It consists of 618 links and 58 zones, and the simulation runs over a time horizon from 08:00AM to 09:00AM in 3 periods of 20 minutes. The network used is shown in Figure 1(b). As explained above, the computational experiments are synthetic in nature and use a Ground Truth OD Matrix $\left(\boldsymbol{X}^{\boldsymbol{G T}}\right)$ to generate the input data. Thus, the counts for the 80 most used links are taken as the real counts in the network. In Figure 1(c), the detection layout is coloured in orange once an assignment is made to the network. 

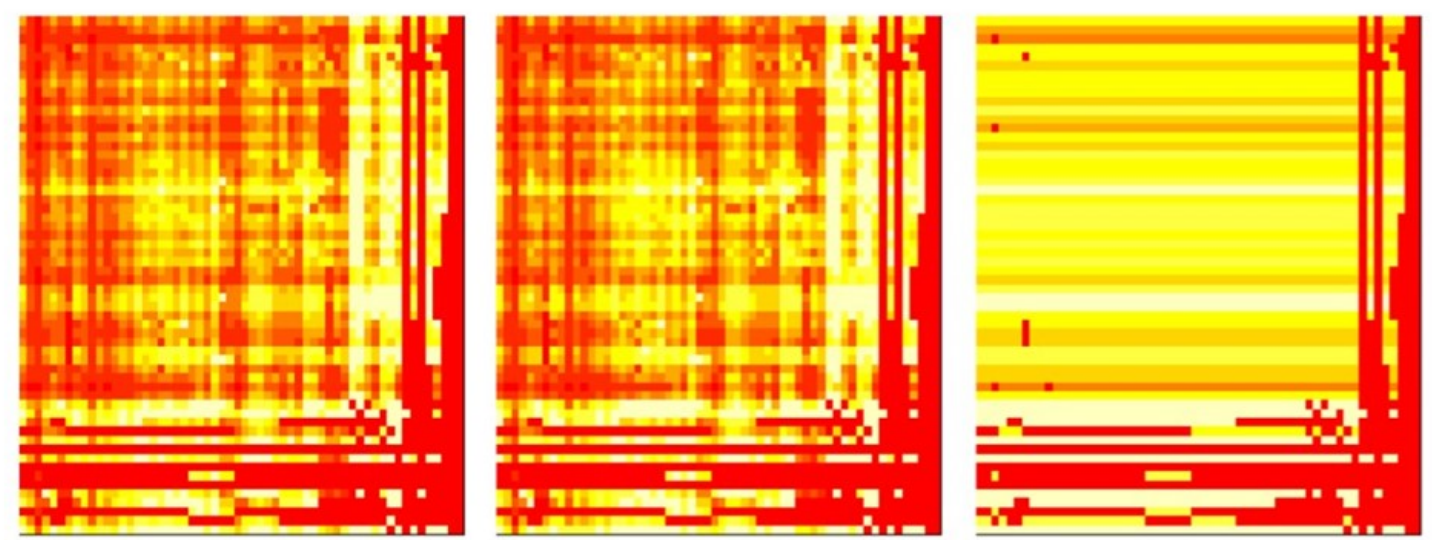

(a)

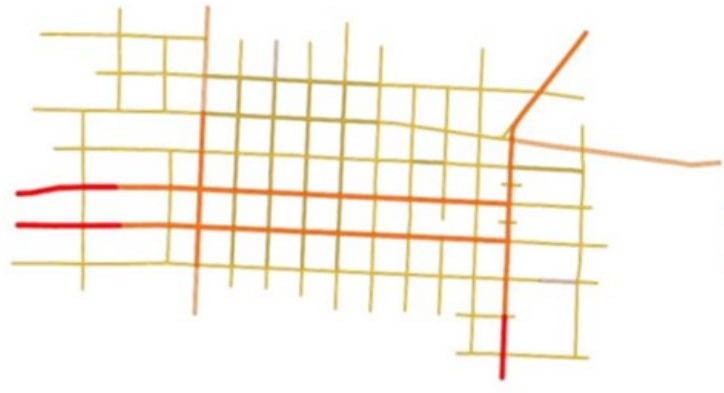

(b)

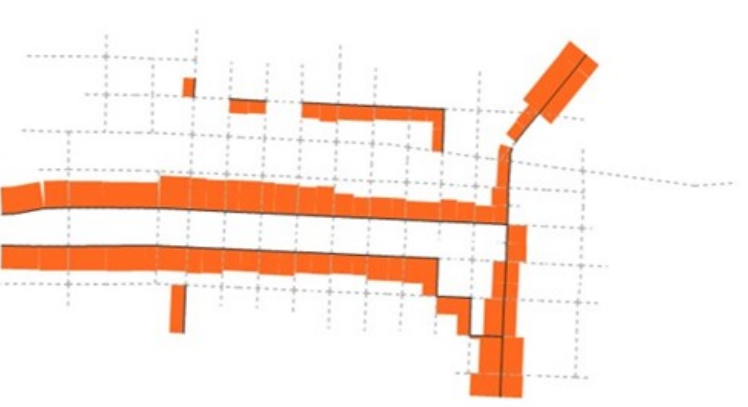

(c)

Figure 1.(a) Heatmaps of the Ground Truth, Incremental+ and Chaos+Inc+. (b)Network of the city of Hillsboro. (c)Detection Layout schema.

\subsection{Measures of quality}

Traditionally, almost all papers on OD matrix estimation determine the quality of the results by using the convergence of the Objective Function and the $R^{2}$ fit between real and simulated traffic counts. From the optimization point of view, these measures are a good selection because they can show explicitly that the method used works specifically for the purpose of minimizing the objective function designed as an OD matrix estimation problem. Furthermore, it verifies that the estimated OD acceptably replicates the observed flows. However, these papers usually do not pay any attention to the quality of the results from a structural point of view. In other words, they 
do not distinguish between whether the traffic OD patterns resulting from their adjustment approach exhibit an acceptable degree of structural similarity to the target matrix or, alternatively, their approach provides a perturbed matrix that is structurally different. If this is the case, then it could be doubtful that such a structural change could be physically interpretable in terms of the underlying transportation system, particularly when taking into account increases or decreases in the total number of trips.

Classical distances between vectors can be applied to matrices by considering both matrices $\boldsymbol{M}, \boldsymbol{N} \in \mathcal{M}_{n}(\mathbb{R})$ as vectors of $\boldsymbol{M}, \boldsymbol{N} \in \mathbb{R}^{n \times n}$. Then, Euclidean, Manhattan and other vector distances can be used in the second component of the OD estimation problem, which is formulated as a minimization problem. However, these distances do not capture the differences and similarities of many aspects, such as the structure of the OD matrix; therefore, the spatiotemporal similarities of the OD matrices are not captured by these measures (Djukic 2014). It seems clear that alternatives to these vector measures must be taken into account when comparing OD matrices.

This measurement is borrowed from the Image Quality Assessment process for comparing two different images. (Wang et al. 2004) presents SSIM - the Structural SIMilarity index - for a matrix of pixels that is the product of three different comparison components: luminance, contrast and structure. Luminance corresponds to the intensity of illumination, which is indeed the mean of the different pixels in a sub-matrix. Contrast is the root of the squared average between pixels once the luminance is removed from the sub-matrix, thus making it the standard deviation. And finally, the structure is compared by using the covariance between the two matrices. These three factors are firstly transformed with the aim of adjusting them to the interval $[0,1]$, where 1 means perfect match and 0 means totally different. SSIM is therefore a 
similarity measure that is independent of the magnitude of the values in the matrix. The formula summarizing this explanation is below:

$$
\operatorname{SSIM}(\boldsymbol{x}, \boldsymbol{y})=l(\boldsymbol{x}, \boldsymbol{y})^{\alpha} c(\boldsymbol{x}, \boldsymbol{y})^{\beta} s(\boldsymbol{x}, \boldsymbol{y})^{\gamma}
$$

where luminance, contrast and structure are defined as:

$$
\left\{\begin{array}{l}
l(\boldsymbol{x}, \boldsymbol{y})=\frac{2 \mu_{x} \mu_{y}+C_{1}}{\mu_{x}^{2}+u_{y}^{2}+C_{1}} \\
c(\boldsymbol{x}, \boldsymbol{y})=\frac{2 \sigma_{x} \sigma_{y}+C_{2}}{\sigma_{x}^{2}+\sigma_{y}^{2}+C_{2}} \\
s(\boldsymbol{x}, \boldsymbol{y})=\frac{\sigma_{x y}+C_{3}}{\sigma_{x} \sigma_{y}+C_{3}}
\end{array}\right.
$$

and $\mu_{x}, \sigma_{x}, \mu_{y}, \sigma_{y}, \sigma_{x y}$ are the mean, standard deviation and covariance of the vectors $\boldsymbol{x}$ and $\boldsymbol{y}$. $C_{1}, C_{2}, C_{3}$ are stability constants for avoiding numerical problems and are typically set to $C_{1}=$ $C_{2}=2 \cdot C_{3}=1$; and $\alpha, \beta, \gamma$ are weighting coefficients that are typically set to 1 (Wang et al. 2004). In image comparison, the MSSIM is computed as the mean of the SSIM of all the submatrices of dimension $\mathrm{N}$, because pixel proximity is crucial in image pattern recognition.

In the case of OD matrices, MSSIM is very useful, as suggested in (Djukic 2014). The MSSIM measure uses sliding boxes that correspond to the submatrices of the compared matrices in order to estimate its value. (Djukic 2014) conducts a primary analysis of the influence of these sliding boxes in the quality of the results. (Behara, et al. 2018) conducts a complementary analysis to determine operational rules for selecting boxes that are not easily applicable. This paper proposes a more meaningful variant that is easy to apply in practice, consisting of calculating the averages according to rows and columns rather than sub-matrices, that is, by using rectangular sliding rules that correspond to either rows or columns in the OD matrix. One row in an OD matrix represents the distribution of trips departing from a single origin zone 
while, analogously, one column is the distribution of trips arriving at a single destination zone. This therefore corresponds to a physical interpretation of patterns in the underlying transport system. Thus, SSIM will capture the similarity between these described distributions by considering the mean, the variance and the structure of departing and arriving distributions, all of which correspond to the structural property of the trip patterns described by the OD.

\section{Computational experiments}

These experiments address different situations, from similar-structure matrices with different numbers of trips to non-similar-structure matrices. Thus, all 6 initializations are considered for both procedures and their variants.

\subsection{Dynamic Spiess approach}

The three versions of Dynamic Spiess discussed in Section 2, have been tested:

- Original Spiess: A Major iteration that assigns the OD matrix $\boldsymbol{X}_{\boldsymbol{k}}$ in order to update the assignment matrix $\boldsymbol{A}_{\boldsymbol{k}}$. This is done after a Minor iteration at the upper level updates the OD matrix.

- Alternative Spiess: Major iterations are done after the upper level optimization converges, when the relative error is lower than $10^{-4}$.

We test these versions using the objective function in Equation (11), with $w=0$ and $w=$ 1, and the quadratic distance function in Section 2.2.2. The Entropy function has been tested only with the Dynamic Original Spiess version, which also uses $w=1$. 
The objective of the experiments is to analyse both the performance of the algorithm and the quality of the solution arrived at by the OD estimation procedure. Figure 2 shows the results of the Dynamic Original Spiess version in 4 different experiments. As seen in Figure 2(a), the descent of the objective function is fast and stable. The quality of the results is usually assessed in terms of the correlation between the measured link flows $\widehat{\boldsymbol{Y}}$ and the estimated link flows $\boldsymbol{Y}$, which are provided by the matrix $\boldsymbol{X}$ that results from the adjustment and the plot in Figure 2(b). The evolution of the Total Number of Trips is plotted in Figure 2(c), which does not correctly approximate the Ground Truth total number of trips ( $N T=9,878$ trips), which is incremented in all cases.
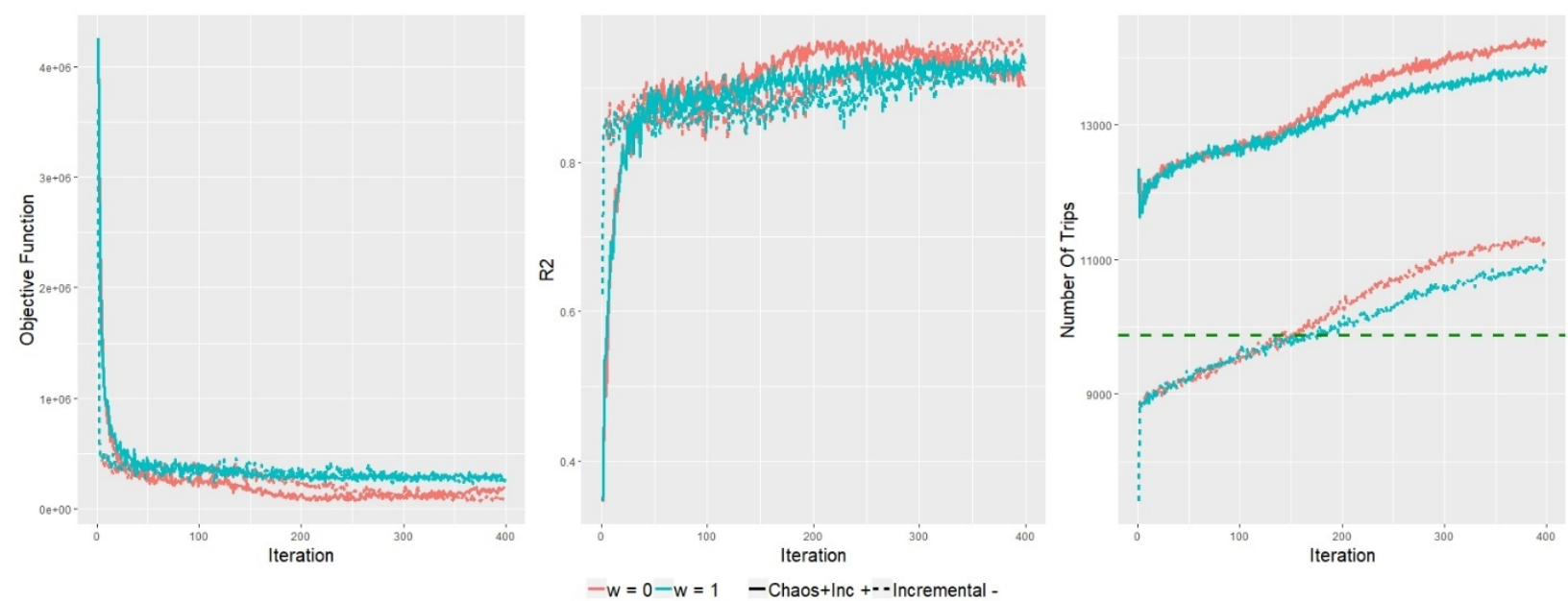

Figure 2.Results for Dynamic Original Spiess for different initializations: (a) Objective Function (b) $R^{2}$ (c) Num-Of-Trips.

In most cases, the correlation coefficient becomes large enough to consider the result significantly good, and both the fast convergence and the high values of $R^{2}$ allow finding an adjusted OD matrix in just a few iterations. However, more detailed insight reveals that this cannot be the case, since the adjustment process behaves as a meta-regression model that 
balances flows among the implied OD pairs surveyed by the detection layout, while its action on other parts of the network, for which no additional information is available, could be rather undefined. Table 1 presents the results of the set of experiments (with all the different initializations) according to which adjusted matrices would be acceptable. $R_{0}^{2}$ and $R_{f}^{2}$ are the fittings between the real counts and the simulated counts before and after the OD estimation process. $M S S I M_{0}$ and $\operatorname{MSSIM}_{f}$ stand for the MSSIM measure before and after the process of the OD matrix with respect to the Ground Truth OD matrix. The basic number of trip indicators has also been added in order to analyse the Dynamic Spiess proposal. Some conclusions are stated:

- The Dynamic Spiess estimation procedure seems to work as a meta-regression procedure in which $R_{f}^{2}$ is high no matter the initial matrix. However, the structural similarity, $\operatorname{MSSIM}_{f}$, shows that the quality of the outcome does not correspond to the $R_{f}^{2}$ fit.

- The use of a distance term with respect to the initial matrix $\boldsymbol{X}^{\boldsymbol{H}}$ generally has a slightly negative effect on $R_{f}^{2}$. But in these cases, MSSIM values are higher when the seed OD matrices are similar to the GT OD matrix.

- The indicator for the number of trips does not converge to the GT matrix value $(9,878$ trips), but instead increments in all cases the number of trips added to the network. Slightly more promising results are obtained when the distance function to the seed OD matrix is added to the Objective Function.

- Regarding the experiments conducted with less computational effort by launching the assignment only when the optimization engine converged, the $R_{f}^{2}$ final values are not as high as those in the other experiments, and the total number of trips increases more. That 
fact confirms the importance of treating the DODE problem as a bilevel optimization problem that, at each iteration, can access the lower level in order to launch a complete Traffic Assignment.

- The results obtained with the entropy function and $w=1$ are similar to those using the Euclidean distance, and they present no evidence of better performance when the second term is added to the objective function.

The results when $w=1$ show slightly better performance with respect to the Number of Trips and MSSIM than when $w=0$, which tends to outperform the former in terms of $R_{f}^{2}$. Furthermore, adding the second term also adds to the computational burden. Therefore, the term indicating the distance to the initial matrix will not be considered in the following experiments, which we conduct using our new approach based on simulation optimization techniques.

\subsection{SPSA variants with only Traffic Counts}

The original SPSA described above has been tested. The objective function used in these cases is the same as in the Dynamic Spiess procedure, Equation (6), with the aim of comparing one descent to the other without the distance term to the seed OD matrix. $n_{g}=5$ has been selected, based on (Kostic et al. 2017), having into account the equilibrium between a large number and the computational effort of the procedure.

SPSA variants adding transport information have been generically tested for the test site. Based on the historical OD matrix for each experiment, constraints of the form $(1-\beta) \boldsymbol{X}^{\boldsymbol{H}} \leq$ $\boldsymbol{X} \leq(1+\beta) \boldsymbol{X}^{\boldsymbol{H}}$ have been introduced with $\beta=0.25$. This means that the OD values can vary between $-25 \%$ and $+25 \%$ of their original values, which come from a historical and reliable OD 
matrix. In this section, the three variants are compared and will be called Free SPSA, Constrained SPSA and Penalized SPSA. 

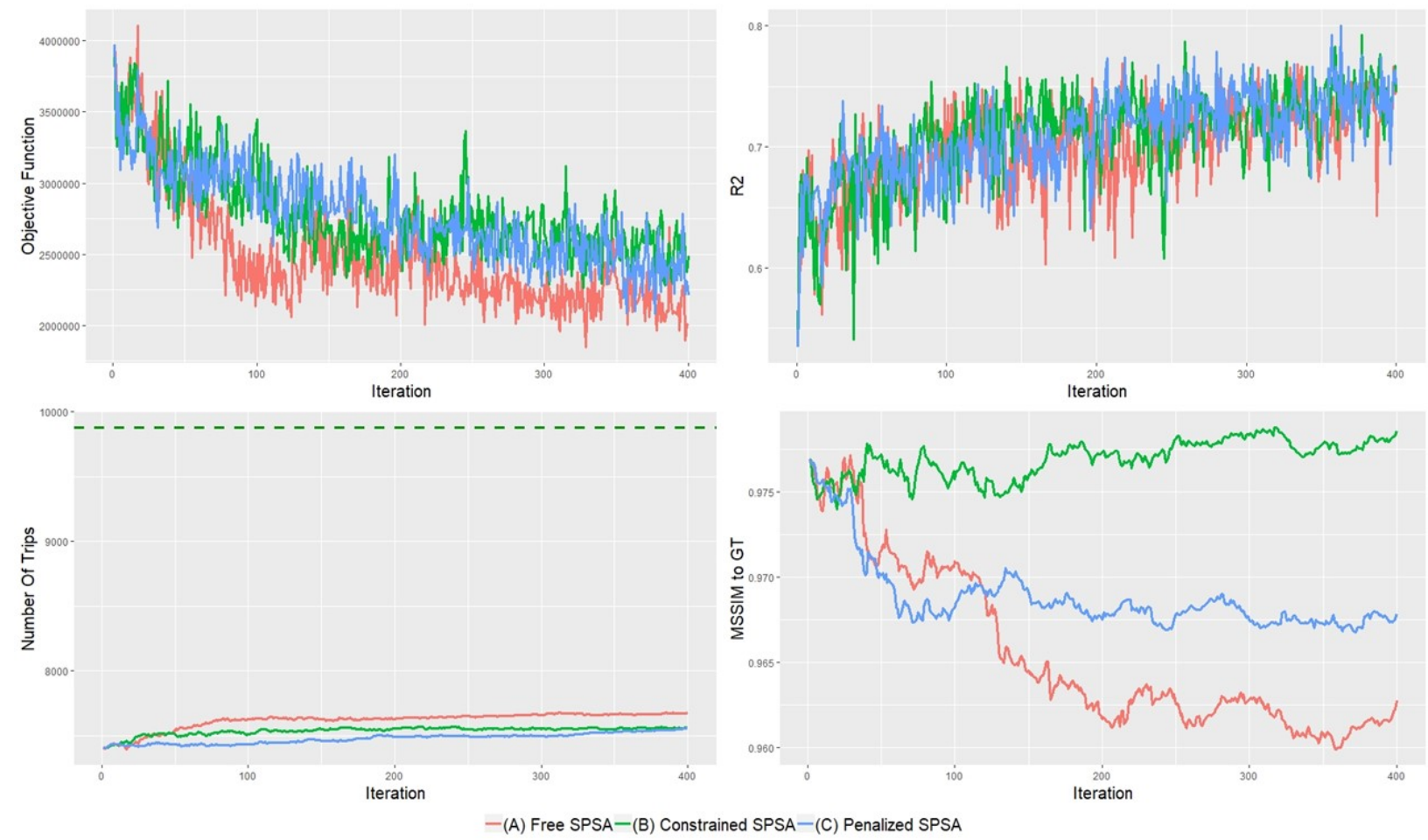

(a)
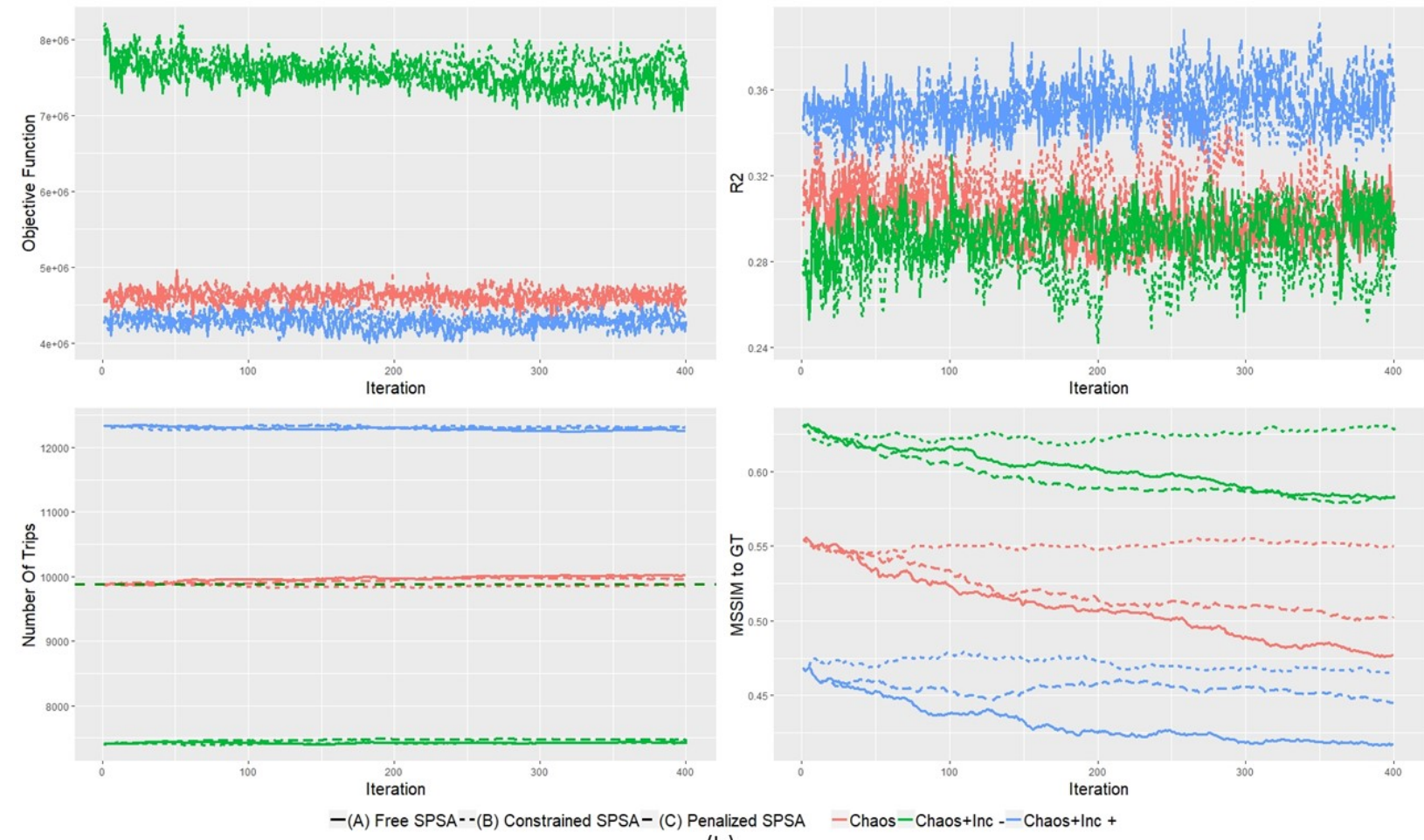

(b)

Figure 3:(a) Results for the different variants of SPSA (without CG) and Multitude initial OD matrix. (b) Results for SPSA without Conjugate Gradient for the three structurally different 
initializations [(i) Objective Function. (ii) $R^{2}$. (iii) Num-Of-Trips. (iv) MMSIM with respect to the GT OD Matrix].

Figure 3(a) shows the behaviour of the three different variants of SPSA with a same initial OD matrix. Figure 3(a-i) and (a-ii) show the acceptable performance of all SPSA variants as a minimization procedure and as a metaregressor for traffic flow counts. In these cases, the descent is clear, despite the oscillations coming from the stochastic nature of the algorithm. The Total Number of Trips, Figure 3(a-iii), is unable to increase significantly to approach the Ground Truth Total Number of Trips.

Naturally, the Free SPSA presents a better descent, followed by Penalized SPSA and, finally, the Constrained SPSA, which is the one with the worst descent. A similar phenomenon occurs in the evolution of the Total Number of Trips, where Free SPSA is the one that most approximates the Ground Truth OD matrix while Penalized and Constrained SPSA approximate it less because of the constraints added to the problem. On the other hand, the constraints added to the problem are reflected on the MSSIM evolution where clearly these constraints help to maintain the structure of the OD matrix, more similar to the GT OD matrix structure. Constrained SPSA seems to perform better.

A comparison between all plots in Figure 3(a) and Figure 3(b) shows clearly that SPSA variants work properly only for those initial OD matrices that are structurally similar to the Ground Truth OD matrix. When the seed OD matrix is one of the Chaos initializations (Figure 3(b)), the SPSA variants do not act as a minimization procedure; they do not increase $R^{2}$ values; and they do not perturb the matrices at all. The variants are therefore useless in these cases, indicating that the SPSA procedures are in no way robust in terms of the initial OD matrix. This 
is a drawback to using SPSA when one is not confident about the structure of the seed OD matrices being used.
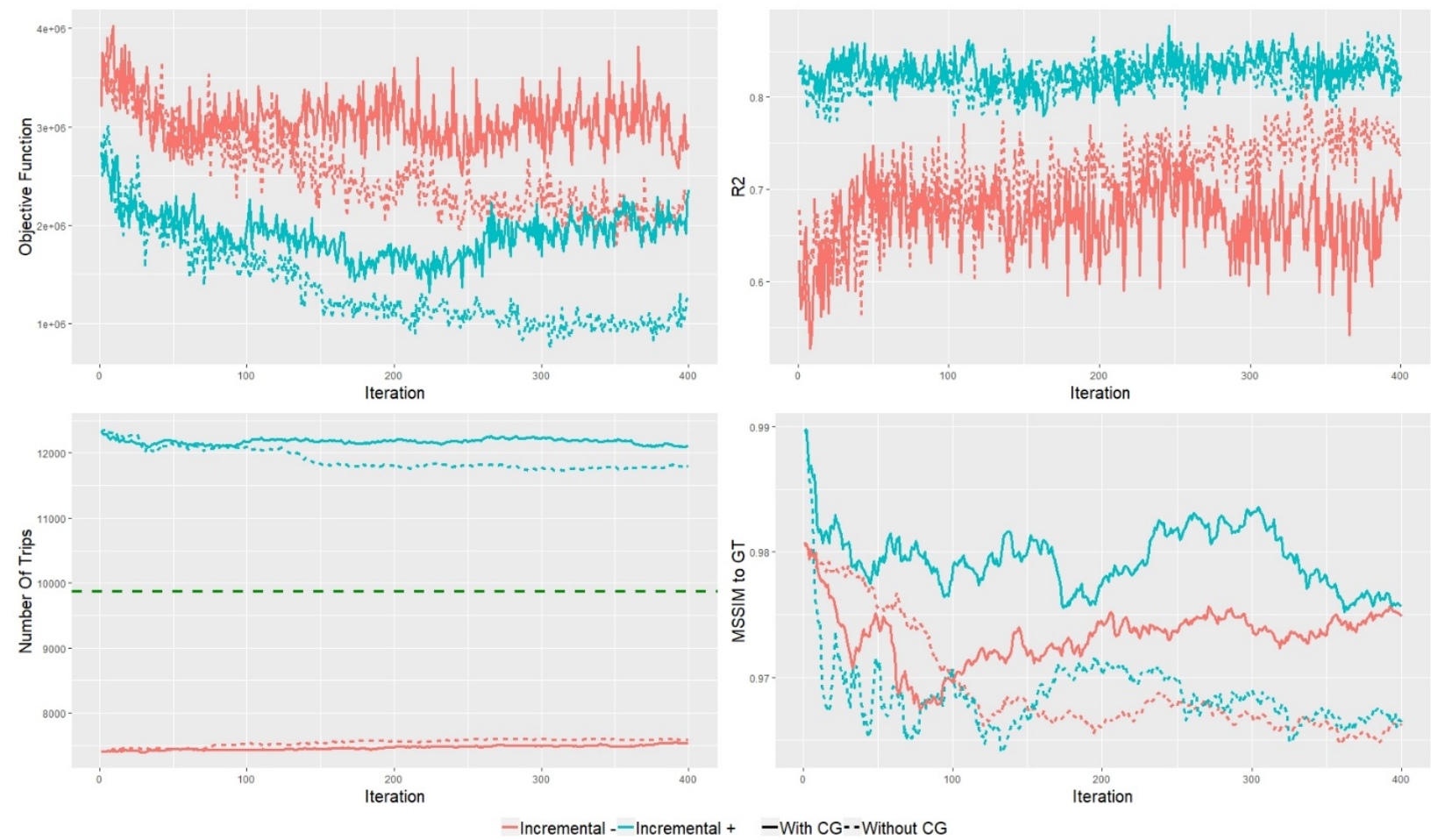

Figure 4: Results for the Constrained SPSA with and without Conjugate Gradient for two initializations: (a) Objective Function. (b) $R^{2}$. (c) Num-Of-Trips. (d) MMSIM with respect to the GT OD Matrix.

Figure 4 shows that the Conjugate Gradient version in the SPSA does not perform better for the different initializations.

Finally, Table 1 summarizes the final results for all the whole set of experiments using SPSA variants and the 6 different initial OD matrices. It confirms that, in general, the results with the Conjugate Gradient variant are worse than those without it. The same observations regarding the figures above can be concluded from the table: When the initial OD matrix is 
similar to the Ground Truth OD matrix, and according to the $M S S I M_{0}$ indicator, SPSA reaches a reasonably acceptable adjusted OD matrix that always presents a gap according to the Total Number of Trips. This total remains close to the historical OD matrix's number of trips, which provides only a slightly smooth approximation. On the other hand, when the seed OD matrix presents another mobility pattern (or matrix structure), SPSA is not able to correctly adjust the OD matrix. 
Table 1. Results for the OD matrix estimation using the different methods

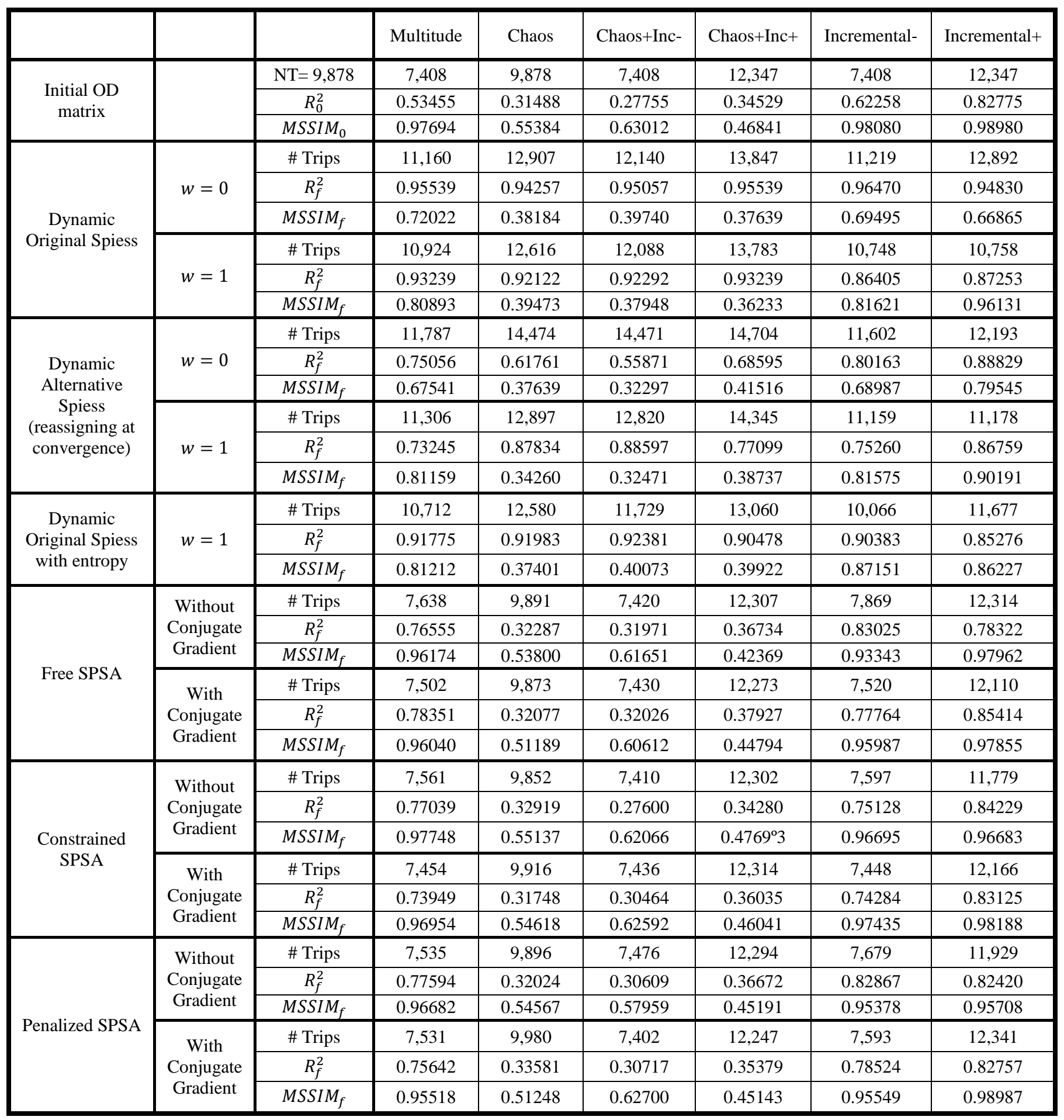




\section{Conclusions}

The experimental framework for the synthetic computational experiments assumes the a priori knowledge of a Ground Truth OD matrix, $\boldsymbol{X}^{\boldsymbol{G T}}$, from which a set of link flow measurements $\widehat{\boldsymbol{Y}}$ has been generated for a subset of links $l \in \widehat{L} \subseteq L$ in the network, taken as the real measurements. The Ground Truth OD matrix $\boldsymbol{X}^{\boldsymbol{G T}}$ has a total number of trips $N^{G T}$.

The hypothetical Ground Truth OD matrix $\boldsymbol{X}^{\boldsymbol{G T}}$ has been perturbed, as described in Section 4.1, to generate various historical OD matrices $\boldsymbol{X}^{\boldsymbol{H}}$, each with a number of trips $N^{H}$, to be used as initial matrices in the computational experiments. Each computational experiment provides an estimate $\boldsymbol{X}$ of the adjusted or corrected OD matrix, with a total number of trips $N^{X}$, and a set $\boldsymbol{Y}$ of estimated link flows for the subset of links $l \in \hat{L} \subseteq L$ in the network.

The computational results obtained using the Dynamic Spiess method are shown in Table 1 and can be summarized as follows:

- The dynamic version of (Spiess 1990), implemented with the Simulation-based Assignment of Visum18, shows an excellent convergence behavior in all cases.

- If the quality of the resulting adjusted OD matrix $\boldsymbol{X}$ is measured, as usually, in terms of the regression between the measured flows $\widehat{\boldsymbol{Y}}$ and the estimated flows $\boldsymbol{Y}$, the conclusion is that the Dynamic Spiess Algorithm has a very good performance and that any of the estimated $\boldsymbol{X}$ matrices reproduces the link flows very well.

- But, not all the resulting $\boldsymbol{X}$ are of the same quality:

o The comparison of $N^{G T}$ with $N^{X}$ shows significant discrepancies depending on the kind of perturbation with which $\boldsymbol{X}^{\boldsymbol{H}}$ was generated.

o The structural similitude analysis in terms of MSSIM between $\boldsymbol{X}^{\boldsymbol{G T}}$ and $\boldsymbol{X}$ shows: 
- An acceptable degree of structural similitude when the perturbation generating $\boldsymbol{X}^{\boldsymbol{H}}$ produces a seed OD matrix roughly close to $\boldsymbol{X}^{\boldsymbol{G T}}$.

- A significant structural dissimilitude when $\boldsymbol{X}^{H}$ is significantly far from $X^{G T}$.

o Therefore one can conclude that, although the new analytical approach exhibits nice convergence properties - in terms of rapidly reducing the value of the objective function to a stable result - and shows a high capacity of reproducing the observed flows $\widehat{\boldsymbol{Y}}$, it is unable to identify the hypothetical Ground Truth $\boldsymbol{X}^{\boldsymbol{G T}}$, or a matrix close to $\boldsymbol{X}^{G T}$ with a similar structure, when the seed matrix is rather different from it.

The weighting factor $w_{1}$ for the distance term $F_{1}\left(\boldsymbol{X}, \boldsymbol{X}^{\boldsymbol{H}}\right)$ between the OD matrices set to zero, $w_{1}=0$, have been considered. While the results with $w_{1} \neq 0$ show a slightly better performance, although an increase of the computational burden. These results look similar to other researchers, (Toledo and Kolechkina 2013), which allocate a negligible weight to this term of the objective function. When the initializations correspond to perturbed matrices, structurally very different, from the GT OD matrix, the analytical approaches exhibit a degree of robustness leading to a relatively close solution. While the SPSA seems to get lost converging only when the initial values are not that far.

This paper has explored the SPSA simulation-based approach, for its intrinsic capability to include new terms in the objective function. However, before testing the extensions of SPSA, it should be verified the quality of the solutions provided and its performance. The computational results, shown in Table 1, indicate that: 
- The conventional SPSA has a worse convergence behavior than the analytical approaches. If the quality of the resulting adjusted OD matrix $\boldsymbol{X}$ is measured, in terms of the regression between the measured flows $\widehat{\boldsymbol{Y}}$ and the estimated flows $\boldsymbol{Y}$, then the results are not as good as those for the analytical approach. Furthermore, the relationship between $N^{G T}$ and $N^{X}$ exhibits an anomalous behavior in some cases and, in terms of MSSIM and although they are bounded, $\boldsymbol{X}^{\boldsymbol{G T}}$ and $\boldsymbol{X}$ show significant differences. The results are more acceptable when $\boldsymbol{X}^{\boldsymbol{H}}$ is close to $\boldsymbol{X}^{\boldsymbol{G T}}$.

- The proposed improvements to SPSA aimed at limiting the growth of $N^{X}$ while keeping or improving the structural similitudes between $\boldsymbol{X}^{\boldsymbol{G T}}, \boldsymbol{X}^{\boldsymbol{H}}$ and $\boldsymbol{X}$, led to two alternative formulations. First, a constrained SPSA in terms of bounding constraints on the changes in the entries of $\boldsymbol{X}$. Second, a penalized SPSA, adding a penalty term to the objective function to limit the changes in the entries of $\boldsymbol{X}$. Both versions provided similar results in terms of:

o Better convergence behavior than the conventional approaches.

o Measuring the quality of the adjusted OD matrix $\boldsymbol{X}$, in terms of the regression between the measured flows $\left\{\widehat{Y}_{l}\right\}$ and the estimated flows $\left\{Y_{l}\right\}$ for $\forall l \in \hat{L} \subseteq L$, the results are significantly better than those for the conventional approach.

o The relationships between $N^{G T}$ and $N^{X}$ are significantly improved.

o In terms of MSSIM $\boldsymbol{X}^{\boldsymbol{G T}}$ and $\boldsymbol{X}$ are much closer than using Free SPSA.

o The results also seem more acceptable when $\boldsymbol{X}^{\boldsymbol{H}}$ is close to $\boldsymbol{X}^{\boldsymbol{G T}}$.

- However, from a computational point of view the constrained version outperforms the other versions, and seems, therefore, the most appropriate to investigate the open questions raised by this analysis. 


\section{Acknowledgement}

This research was funded by TRA2016-76914-C3-1-P Spanish R+D Programs, Secretaria d’Universitats-i-Recerca-Generalitat de Catalunya-2017-SGR-1749 and Industrial-PhD-Program 2017-DI-041.

\section{References}

Antoniou, Constantinos, Jaume Barceló, Martijn Breen, Manuel Bullejos, Jordi Casas, Ernesto Cipriani, Biagio Ciuffo, Tamara Djukic, Serge Hoogendoorn, Vittorio Marzano, Lídia Montero, Marialisa Nigro, Josep Perarnau, Vincenzo Punzo, Tomer Toledo, and Hans van Lint. 2016. “Towards a Generic Benchmarking Platform for Origin-Destination Flows Estimation/Updating Algorithms: Design, Demonstration and Validation.” Transportation Research Part C 66:79-98.

Antoniou, Constantinos, Carlos Lima Azevedo, Lu Lu, Francisco Pereira, and Moshe BenAkiva. 2015. "W-SPSA in Practice: Approximation of Weight Matrices and Calibration of Traffic Simulation Models.” Transportation Research Part C 59:129-46.

Behara, Krishna N. S., Ashish Bhaskar, and Edward Chung. 2018. "Classification of Typical Bluetooth OD Matrices Based on Structural Similarity of Travel Patterns- Case Study on Brisbane City.” in Transportation Research Board 97th Annual Meeting.

Bullejos, Manuel, Jaume Barceló, and Lídia Montero. 2014. “A DUE Based Bilevel Optimization Approach for the Estimation of Time Sliced OD Matrices.” International Symposium of Transport Simulation 2014 00:1-19.

Cantelmo, Guido, Ernesto Cipriani, Andrea Gemma, and Marialisa Nigro. 2014. “An Adaptive Bi-Level Gradient Procedure for the Estimation of Dynamic Traffic Demand.” IEEE Transactions on Intelligent Transportation Systems 15(3):1348-61.

Carrese, Stefano, Ernesto Cipriani, Livia Mannini, and Marialisa Nigro. 2017. "Dynamic Demand Estimation and Prediction for Traffic Urban Networks Adopting New Data Sources.” Transportation Research Part C 81:83-98.

Cipriani, Ernesto, Michael Florian, Michael Mahut, and Marialisa Nigro. 2011. “A Gradient Approximation Approach for Adjusting Temporal Origin-Destination Matrices.” Transportation Research Part C 19(2):270-82.

Codina, Esteve and Lídia Montero. 2006. “Approximation of the Steepest Descent Direction for the O-D Matrix Adjustment Problem.” Pp.329-62 in Annals of Operations Research. Vol.144. Springer US.

Djukic, Tamara. 2014. "Dynamic OD Demand Estimation and Prediction for Dynamic Traffic Management.” TU Delft. 
Frederix, Rodric, Francesco Viti, Ruben Corthout, and Chris Tampère. 2011. "New Gradient Approximation Method for Dynamic Origin-Destination Matrix Estimation on Congested Networks.” Transportation Research Record: Journal of the Transportation Research Board 2263(1):19-25.

Frederix, Rodric, Francesco Viti, and Chris Tampère. 2013. "Dynamic Origin-Destination Estimation in Congested Networks: Theoretical Findings and Implications in Practice.” Transportmetrica A: Transport Science 9(6):494-513.

Kostic, Bojan, Guido Gentile, and Constantinos Antoniou. 2017. "Techniques for Improving the Effectiveness of the SPSA Algorithm in Dynamic Demand Calibration.” Pp. 368-73 in 5th IEEE International Conference on Models and Technologies for Intelligent Transportation Systems, MT-ITS 2017 - Proceedings. IEEE.

Lu, Lu, Yan Xu, Constantinos Antoniou, and Moshe Ben-Akiva. 2015. “An Enhanced SPSA Algorithm for the Calibration of Dynamic Traffic Assignment Models.” Transportation Research Part C 51:149-66.

Luenberger, David G. and Yinyu Ye. 1973. Linear and Nonlinear Programming. Vol.228.

Lundgren, Jan T. and Anders Peterson. 2008. "A Heuristic for the Bilevel Origin-DestinationMatrix Estimation Problem.” Transportation Research Part B: Methodological 42(4):339_ 54.

Nigro, Marialisa, Akmal Abdelfatah, Ernesto Cipriani, Chiara Colombaroni, Gaetano Fusco, and Andrea Gemma. 2018. "Dynamic O-D Demand Estimation: Application of SPSA AD-PI Method in Conjunction with Different Assignment Strategies.” Journal of Advanced Transportation 2018:1-18.

Osorio, Carolina and Linsen Chong. 2015. “A Computationally Efficient Simulation-Based Optimization Algorithm for Large-Scale Urban Transportation Problems.” Transportation Science 49(3):623-36.

PTV AG. 2018. “VISUM.”

Ros-Roca, Xavier, Lídia Montero, and Jaume Barceló. 2017. "Notes on Using SimulationOptimization Techniques in Traffic Simulation.” Pp.881-88 in Transportation Research Procedia. Vol.27.

Ros-Roca, Xavier, Lídia Montero, Arne Schneck, and Jaume Barceló. 2018. "Investigating the Performance of SPSA in Simulation-Optimization Approaches to Transportation Problems.” Transportation Research Procedia 34:83-90.

Sadegh, Payman and James C. Spall. 1998. “Optimal Random Perturbations for Stochastic Approximation Using a Simultaneous Perturbation Gradient Approximation.” IEEE Transactions on Automatic Control.

Spall, James C. 1992. "Multivariate Stochastic Approximation Using a Simultaneous 
Perturbation Gradient Approximation.” IEEE Transactions on Automatic Control.

Spall, James C. 1998. "An Overview of the Simultaneous Perturbation Method for Efficient Optimization.” Johns Hopkins APL Technical Digest (Applied Physics Laboratory).

Spall, James C. 2003. Introduction to Stochastic Search and Optimization: Estimation, Simulation, and Control. Wiley-Interscience.

Spiess, Heinz. 1990. “A Gradient Approach for the OD Matrix Adjustment Problem.” Centre for Research on Transportation, University of Montreal, Canada 693(Publication No. 693, CRT):1-11.

Toledo, Tomer and Tanya Kolechkina. 2013. "Estimation of Dynamic Origin-Destination Matrices Using Linear Assignment Matrix Approximations.” IEEE Transactions on Intelligent Transportation Systems 14(2):618-26.

Tympakianaki, Athina, Haris N. Koutsopoulos, and Erik Jenelius. 2015. "C-SPSA: Cluster-Wise Simultaneous Perturbation Stochastic Approximation Algorithm and Its Application to Dynamic Origin-Destination Matrix Estimation.” Transportation Research Part C 55:23145.

Wang, I. Jeng and James C. Spall. 1999. "A Constrained Simultaneous Perturbation Stochastic Approximation Algorithm Based on Penalty Functions.” Pp.393-99vol.1 in Proceedings of the 1999 American Control Conference (Cat.No.99CH36251). IEEE.

Wang, Z., A. C. Bovik, H. R. Sheikh, and E. P. Simoncelli. 2004. "Image Quality Assessment: From Error Visibility to Structural Similarity.” IEEE Transactions on Image Processing 13(4):600-612.

Yang, Xianfeng, Yang Lu, and Wei Hao. 2017. "Origin-Destination Estimation Using Probe Vehicle Trajectory and Link Counts.” Journal of Advanced Transportation 2017:1-18. 